\title{
Gold-catalyzed post-Ugi alkyne hydroarylation for the synthesis of 2-quinolones
}

\author{
Xiaochen Du ${ }^{\ddagger 1}$, Jianjun Huang ${ }^{\ddagger 1}$, Anton A. Nechaev ${ }^{2}$, Ruwei Yao ${ }^{1}$, Jing Gong ${ }^{1}$, \\ Erik V. Van der Eycken ${ }^{2,3}$, Olga P. Pereshivko*1 and Vsevolod A. Peshkov ${ }^{* 1,4, \S}$
}

\author{
Full Research Paper \\ Address: \\ ${ }^{1}$ College of Chemistry, Chemical Engineering and Materials Science, \\ Soochow University, Dushu Lake Campus, Suzhou, 215123, China, \\ 2Laboratory for Organic \& Microwave-Assisted Chemistry (LOMAC), \\ Department of Chemistry, University of Leuven (KU Leuven), \\ Celestijnenlaan 200F, 3001 Leuven, Belgium, ${ }^{3}$ Peoples' Friendship \\ University of Russia (RUDN University), 6 Miklukho-Maklaya street, \\ Moscow, 117198, Russia and ${ }^{4}$ Department of Chemistry, School of \\ Science and Technology, Nazarbayev University, 53 Kabanbay Batyr \\ Ave, Block 7, Astana, 010000, Republic of Kazakhstan \\ Email: \\ Olga P. Pereshivko* - olga@suda.edu.cn; Vsevolod A. Peshkov* - \\ vsevolod@suda.edu.cn \\ * Corresponding author ‡ Equal contributors \\ § Alternative email address: vsevolod.peshkov@nu.edu.kz \\ Keywords: \\ gold catalysis; hydroarylation; 2-quinolones; Ugi reaction \\ Beilstein J. Org. Chem. 2018, 14, 2572-2579. \\ doi:10.3762/bjoc. 14.234 \\ Received: 07 June 2018 \\ Accepted: 13 September 2018 \\ Published: 04 October 2018 \\ Associate Editor: J. Aubé \\ (c) 2018 Du et al.; licensee Beilstein-Institut. \\ License and terms: see end of document.
}

\begin{abstract}
A series of propargylamides containing an electron-rich benzene ring was prepared through the Ugi reaction of 3,5-dimethoxyaniline with various propiolic acids, aldehydes and isocyanides. Subjecting these adducts to a gold-catalyzed intramolecular alkyne hydroarylation process allowed to efficiently construct the 2-quinolone core bearing a branched substituent on the nitrogen atom.
\end{abstract}

\section{Introduction}

Quinoline and its oxidized derivatives, 2-quinolone and 4-quinolone, are the core structural elements of many natural products and pharmaceutical agents [1-3]. In particular, 2-quinolone derivatives show a broad range of biological activities including antiviral [4-7], antimicrobial [8,9], antiparasitic $[10,11]$, anti-inflammatory $[12,13]$ and anticancer [9,13-19]. In addition, 2-quinolones were identified as promising entities for the treatment of neuropathic pain [20-22] and erectile dysfunction [23]. Therefore, the elaboration of practical methodologies for the synthesis [24] and functionalization [25-29] of the 2-quinolone scaffold has become a budding research trend. In the last decade, a great number of efficient approaches has been developed utilizing transition metal-catalyzed [30-32], Lewis acid-mediated [33], and radical cyclizations [34] of various aniline derivatives with most recent strategies focusing on the implementation of transition metal-catalyzed $\mathrm{C}-\mathrm{H}$ activation methods $[35,36]$.

One of the common approaches towards 2-quinolones 2 involves the intramolecular Friedel-Crafts hydroarylation 
[37,38] of $N$-arylamides of 3 -substituted propynoic acids 1 (Scheme 1). It was found that the use of superstoichiometric amounts of strong Brønsted [39-41] or Lewis [40,41] acids give good results on substrates with non-activated $N$-aryl groups such as $\mathbf{1 a}$, while substrate $\mathbf{1 b}$ bearing an additional electrondonating group on the $\mathrm{N}$-aryl fragment gave a poorer outcome under similar settings (Scheme 1a). Consequently, several catalytic methods were developed to extend the scope of this Friedel-Crafts process to substrates such as $\mathbf{1 c}$ and $\mathbf{1 d}$ featuring highly electron-rich $N$-aryl moieties (Scheme 1b) [42-44]. Finally, following the success of the above procedures, two asymmetric versions were designed to provide access to axially chiral 2-quinolone-based heterobiaryls such as $\mathbf{2 e}$ (Scheme 1c) $[45,46]$.

One substantial drawbacks of the previous methodologies is that they do lack an exploration of the substituent diversity on the nitrogen atom. We decided to address this issue by employing propargylamides 7 obtained by a four-component Ugi reaction of propiolic acids 3, aldehydes 4, isocyanides 5 and 3,5-dimethoxyaniline (6a) (Scheme 2). We anticipated that the resulting adducts 7 would readily produce 2-quinolones 8 bearing a branched substituent on the nitrogen atom through an intramolecular gold-catalyzed alkyne hydroarylation reaction (Scheme 2). It should be noted, that heterocyclic syntheses through post-Ugi transformations have been under extensive exploration for the last two decades [47-49], especially in terms of gold-catalyzed Friedel-Crafts-type cyclizations involving attack of electron-rich arenes on the triple bonds, leading to the formation of a great number of fused [50-53] and spirocyclic structures [54-59]. In addition, Ugi adducts have already been successfully utilized for the diversity-oriented synthesis of 2-quinolones using either intramolecular Heck reaction [60] or Knoevenagel condensation $[61,62]$. (a)<smiles>[R]c1cccc(NC(=O)C#Cc2ccccc2)c1</smiles>

1a, $\mathrm{R}=\mathrm{H}$

1b, $\mathrm{R}=\mathrm{MeO}$

2a, $\mathrm{R}=\mathrm{H}$

2b, $\mathrm{R}=\mathrm{MeO}$

A: polyphosphoric acid (125 equiv), $115-120^{\circ} \mathrm{C}$,

$0.5 \mathrm{~h}, 81 \%(2 \mathrm{a})$ [39]

B: $\mathrm{TfOH}$ (37 equiv), $25^{\circ} \mathrm{C}, 100 \mathrm{~h}, 97 \%$ (2a) [40]

TfOH (34 equiv), $20^{\circ} \mathrm{C}, 95 \mathrm{~h}, 40 \%$ (2b) [41]

C: $\mathrm{FSO}_{3} \mathrm{H}$ (25 equiv), $-75^{\circ} \mathrm{C}, 5 \mathrm{~h}, 98 \%(2 \mathrm{a})$ [41]

$\mathrm{D}: \mathrm{AlBr}_{3}$ (5 equiv), DCM, $20^{\circ} \mathrm{C}, 10 \mathrm{~h}, 90 \%$ (2a) [41]

(b)
$1 \mathbf{1 d}, \mathrm{R}=\mathrm{ReO}=\mathrm{H}$<smiles>COc1ccc2ccccc2c1C#CC(=O)N(Cc1ccccc1)c1ccc2ccccc2c1</smiles><smiles>COc1ccc2ccccc2c1-c1cc(=O)n(Cc2ccccc2)c2ccc3ccccc3c12</smiles>

$\mathrm{H}:\left[\mathrm{Pd}\left(\mathrm{CH}_{3} \mathrm{CN}\right)_{4}\right]\left(\mathrm{BF}_{4}\right)_{2}(5 \mathrm{~mol} \%),(S)-x y l-\mathrm{H}_{8}$-binap (6 mol \%), DCE, rt, $72 \mathrm{~h}, 94 \%$ yield, $92 \%$ ee [45]

I: $\mathrm{AuCl}\left(\mathrm{SMe}_{2}\right)(20 \mathrm{~mol} \%), \mathrm{AgBF}_{4}(20 \mathrm{~mol} \%)$, $(R)$-DTBM-Segphos (10 mol \%), DCE, rt, $40 \mathrm{~h}$, $96 \%$ yield, $59 \%$ ee [46]<smiles>CC(C)c1cc(C(C)C)c(-c2ccccc2P)c(C(C)C)c1</smiles>

XPhos<smiles>CCCCC(C)(C)c1ccc2c(c1-c1c(P)ccc3c1CCCC3)CCCC2</smiles>

(S)-xyl- $\mathrm{H}_{8}$-binap $\left(\mathrm{Ar}=3,5-\mathrm{Me}_{2} \mathrm{C}_{6} \mathrm{H}_{3}\right)$<smiles>[R17]c1ccc2c(c1-c1c(P)ccc3c1OCO3)OCO2</smiles>

(R)-DTBM-Segphos

$\left(\mathrm{Ar}=4-\mathrm{MeO}-3,5-t-\mathrm{Bu}_{2} \mathrm{C}_{6} \mathrm{H}_{2}\right)$ 


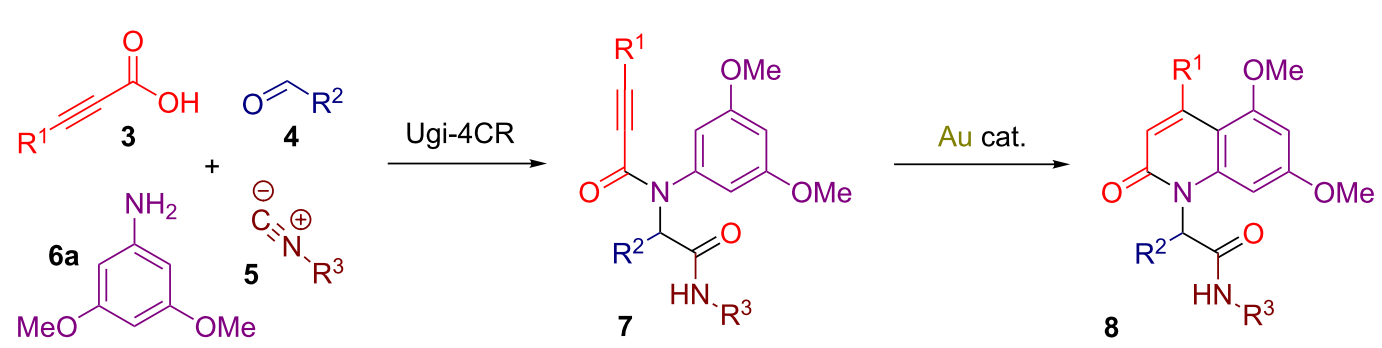

Scheme 2: Strategy towards 2-quinolones 8 bearing a branched substituent on the nitrogen atom.

\section{Results and Discussion}

We began our study with the preparation of the model substrate 7a through the Ugi reaction of tetrolic acid (3a), benzaldehyde (4a), tert-butyl isocyanide (5a) and 3,5-dimethoxyaniline (6a). Next, the cycloisomerization of $7 \mathbf{a}$ was investigated in order to identify the optimal conditions. At first, we attempted two reactions using $5 \mathrm{~mol} \%$ of the standard $\mathrm{AuPPh}_{3} \mathrm{Cl} / \mathrm{AgOTf}$ precatalytic combination in conventional chlorinated solvents such as deuterated chloroform and dichloromethane (Table 1, entries 1 and 2). The latter proved to be a better choice affording the targeted 2-quinolone 8a in up to $90 \%$ yield (Tabel 1, entry 2). Using AgOTf in the absence of gold slowed down the reaction leading to decreased yields of $\mathbf{8 a}$ even at an elevated temperature of $60{ }^{\circ} \mathrm{C}$ (Table 1, entries 3-5). Conducting the $\mathrm{AuPPh}_{3} \mathrm{Cl} /$ AgOTf-catalyzed reaction in trifluoroethanol (TFE) led to im- proved results producing $\mathbf{8 a}$ in up to $97 \%$ yield (Table 1, entry 6). Thus, this greener alternative [63] to chlorinated solvents was selected as the solvent of choice for the further exploration. Changing the silver counterpart to $\mathrm{AgPF}_{6}$ did not affect the reaction outcome yielding $8 \mathrm{a}$ in $96 \%$ (Table 1, entry 7). Using AgOTf or $\mathrm{AuPPh}_{3} \mathrm{Cl}$ as sole catalyst did not provide satisfactory results (Table 1, entries 8-10). In particular, the AgOTfcatalyzed reaction conducted at $60{ }^{\circ} \mathrm{C}$ produced substantial amounts of trifluoroethanol adduct 9a (Table 1, entry 8), while the $\mathrm{AuPPh}_{3} \mathrm{Cl}$-catalyzed reaction suffered from a slow conversion rate of starting $7 \mathbf{a}$ (Table 1, entries 9 and 10).

Next, we attempted to delineate the scope of our methodology (Figure 1). Exploring different 3 -substituted propiolic acids $\mathbf{3}$ in combination with $4 \mathbf{a}, 5 \mathbf{a}$ and $\mathbf{6 a}$ gave moderate results for the

Table 1: Screening of the conditions for the intramolecular hydroarylation of the Ugi adduct $7 \mathbf{a}^{\mathrm{a}}$

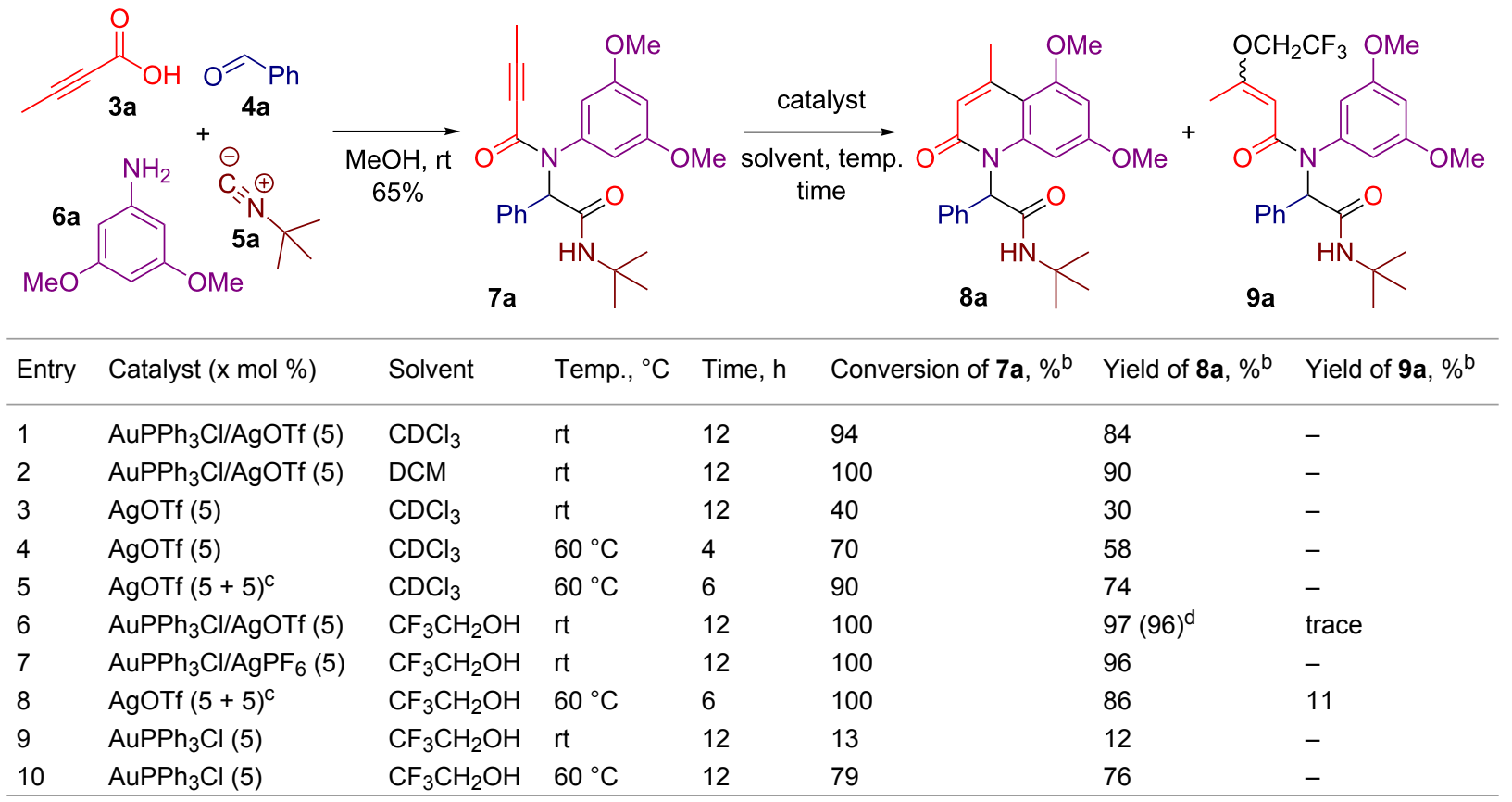

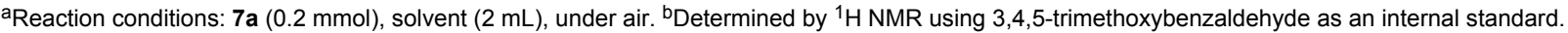
${ }^{\mathrm{c}}$ The first portion of catalyst was added at the beginning of reaction, while the second portion was added after $3 \mathrm{~h}$. ${ }^{\mathrm{d}}$ Isolated yield for a $0.5 \mathrm{mmol}$ scale reaction is given in parenthesis. 

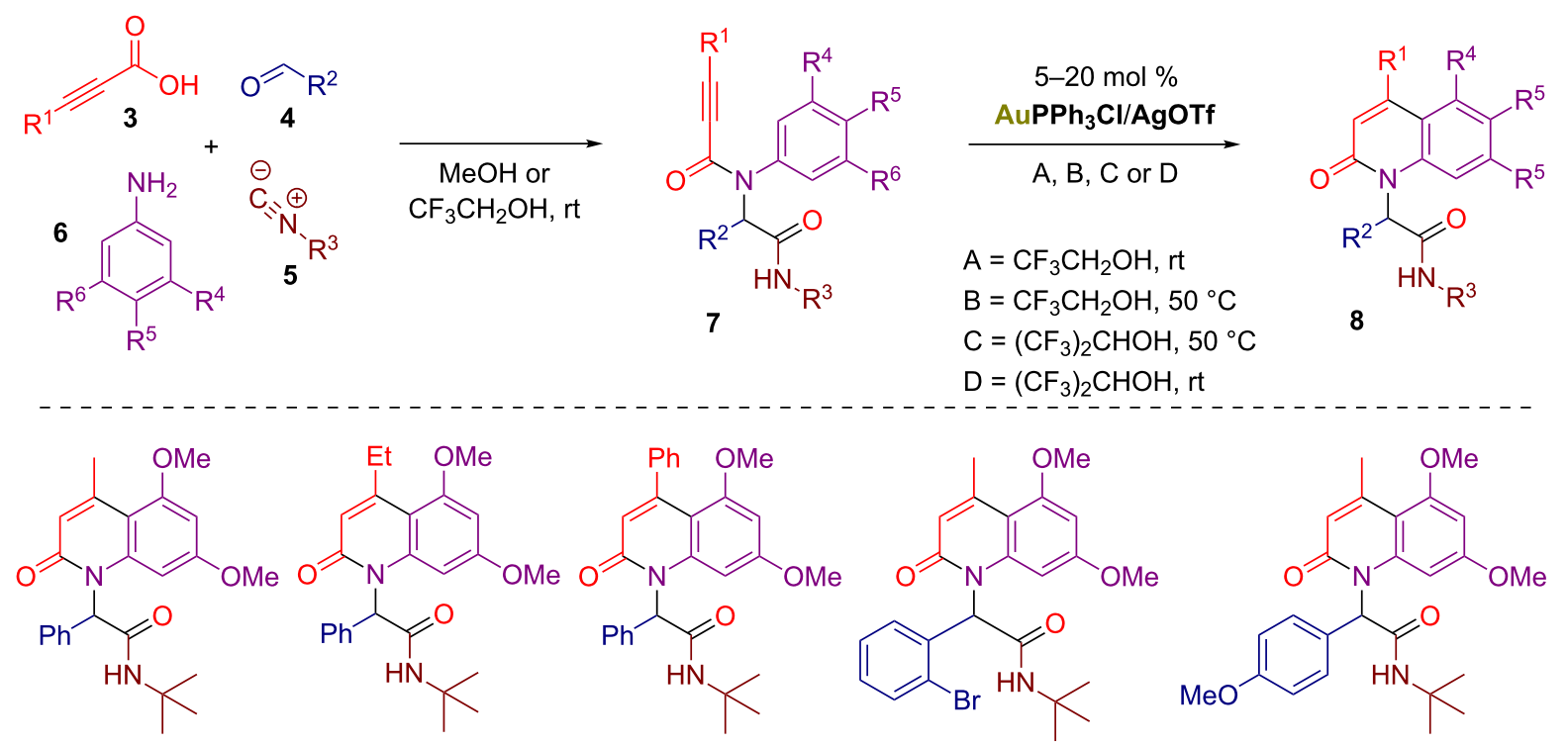<smiles>COc1cc(OC)c2c(c1)c(C)cc(=O)n2C(C(=O)NC(C)(C)C)c1ccccc1Br</smiles>

$8 d, 93 \%(B)$

7d, $67 \%$<smiles>COc1ccc(C(C(=O)NC(C)(C)C)n2c(=O)cc(C)c3c(OC)cc(OC)cc32)cc1</smiles>

(B)

7c, $62 \%$
8 e, $96 \%(B)$

7 e, $83 \%$<smiles>COc1cc(OC)c2c(c1)c(=O)n(C(C(=O)NC(C)(C)C)c1ccc([N+](=O)[O-])cc1)c1cc(OC)cc(OC)c21</smiles>

$8 \mathrm{~h}, 90 \%(\mathrm{~A})$

7h, $29 \%$<smiles>COc1cc(OC)c2c(C)cc(=O)n(C(C(=O)NC(C)(C)C)c3ccc(C(F)(F)F)cc3)c2c1</smiles>

8f, $93 \%(A)$

7f, $32 \%$<smiles>COc1cc(OC)c2c(C)cc(=O)n(C(C(=O)NC(C)(C)C)c3ccc(C#N)cc3)c2c1</smiles>

8g, 92\% (A)

$7 \mathrm{~g}, 26 \%$<smiles>COc1cc(OC)c2c(C)cc(=O)n(C(C(=O)NC(C)(C)C)C3CCCCC3)c2c1</smiles><smiles>COc1cc(OC)c2c(C)cc(=O)n(C(C(=O)NC(C)(C)C)C3CC3)c2c1</smiles><smiles>CCCCNC(=O)C(c1ccccc1)n1c(=O)cc(C)c2c(OC)cc(OC)cc21</smiles>

8j, 94\% (B) 7j, $48 \%$

8k, 99\% (A)

7k, $54 \%$

8I, 97\% (A)

7I, $56 \%$<smiles>COc1cc2c(c(C)cc(=O)n2C(C(=O)NC(C)(C)C)c2ccccc2)c(OC)c1OC</smiles>

$80,89 \%$ (B)

7o, $96 \%$<smiles>Cc1cc(=O)n(C(C(=O)NC(C)(C)C)c2ccccc2)c2cc3c(cc12)OCCO3</smiles>

8p, 58\% (C)

7p, $92 \%$<smiles>COc1cc2c(cc1Br)c(C)cc(=O)n2C(C(=O)NC(C)(C)C)c1ccccc1</smiles>

$8 \mathbf{8}, 75 \%$ (C)

7q, $66 \%$

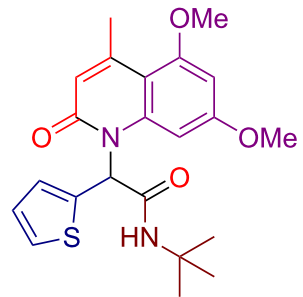

8i, 94\% (A)

$7 i, 48 \%$<smiles>COc1cc(OC)c2c(C)cc(=O)n(C(C(=O)Nc3ccc(C)cc3)c3ccccc3)c2c1</smiles>

$8 \mathrm{~m}, 89 \%(\mathrm{~A})$<smiles>COc1cc(OC)c2c(C)cc(=O)n(C(C(=O)Nc3ccc4ccccc4c3)c3ccccc3)c2c1</smiles>

$8 \mathrm{n}, 83 \%$ (A)

$7 \mathrm{~m}, 77 \%$

7n, $57 \%$

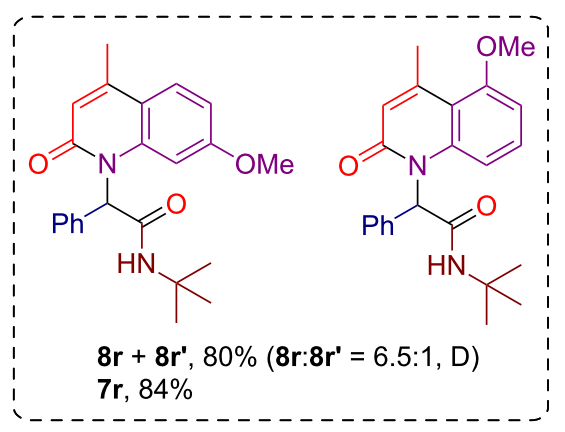


Ugi reaction and good to excellent efficiency for the gold-catalyzed cyclization (Figure 1, products $\mathbf{8 a}-\mathbf{c}$ ). As for the aldehyde component $\mathbf{4}$, both (hetero)aromatic and aliphatic ones were found to be tolerable (Figure 1, products $8 \mathbf{8}-\mathbf{k}$ ), although for the electron-deficient aromatic aldehydes only low yields were achieved during the Ugi step. Yet, the subsequent gold-catalyzed cyclization worked well delivering the 2-quinolones $\mathbf{8 f - h}$ in up to $93 \%$ yield. Besides, several aliphatic and aromatic isocyanates $\mathbf{5}$ have been successfully employed for the preparation of 2-quinolones 81-n. Finally, we decided to investigate the reactions of Ugi adducts $7 \mathbf{0}-\mathbf{r}$ derived from various electron-rich anilines $\mathbf{6}$. The gold-catalyzed cyclization of 3,4,5-trimethoxyaniline-derived substrate $7 \mathbf{0}$ proceeded at the elevated temperature of $50{ }^{\circ} \mathrm{C}$ producing 2-quinolone 80 in $89 \%$ yield. The cyclizations of $\mathbf{7 p}-\mathbf{r}$ required further optimization of the reaction conditions (Tables 2-4) and were complicated due the formation of regioisomeric products. After all, 2-quinolones $\mathbf{8 p}$ and $\mathbf{8 q}$ were isolated in good yields from gold-catalyzed reactions in hexafluoroisopropanol (HFIP), while 2-quinolone $8 \mathbf{r}$ was obtained as a mixture with isomer 8r'.

As noted above, the intramolecular alkyne hydroarylations with certain Ugi adducts required further adjustment of the reaction conditions. For example, the reaction of substrate $7 \mathbf{p}$ being conducted in TFE at room temperature delivered a complex mixture of regioisomeric 2-quinolones $\mathbf{8 p} / \mathbf{8 p}$ ' and TFE-adduct $\mathbf{9 b}$ (Table 2, entry 1). However, the application of branched fluorinated alcohols as solvent solved the problem of the competing alkoxylation reaction. Thus, no alcohol adducts were formed when the gold-catalyzed cyclization of $\mathbf{7 p}$ was conducted in hexafluoro-2-methylpropan-2-ol or HFIP (Table 2, entries 2-4).
Using the latter solvent a full conversion of $7 \mathbf{p}$ was achieved at $50{ }^{\circ} \mathrm{C}$ within $20 \mathrm{~h}$, allowing to isolate both possible products $8 \mathbf{p}$ and $\mathbf{8 p}$ ' in $58 \%$ and $15 \%$ yields, respectively (Table 2, entry 4). It should also be stressed, that reacting $\mathbf{7 p}$ in chloroform at $\mathbf{r t}$ failed to produce any traces of cyclized products (Table 2, entry 5).

A 4-bromo-3-methoxyaniline-derived Ugi adduct $\mathbf{7 q}$ proved to be the most difficult substrate. The initial attempts to perform an intramolecular alkyne hydroarylation of $\mathbf{7 q}$ in TFE were characterized by a rather slow reaction rate which concomitantly promoted the competing alkoxylation reaction (Table 3, entries 1 and 2). Consequently, we were able to isolate and characterize the corresponding TFE-adduct 9c (Table 3, entry 2). Switching to HFIP as the solvent prevented the alkoxylation but led to an even slower reaction rate (Table 3, entries 3-5). Increasing the catalyst loading to 10 and finally up to $20 \mathrm{~mol} \%$ allowed to achieve a full conversion of $\mathbf{7 q}$ within $30 \mathrm{~h}$ producing 2-quinolone $\mathbf{8 q}$ in $75 \%$ isolated yield (Table 3 , entries 5 and $6)$.

Analogously to $\mathbf{7 p}$ and $\mathbf{7 q}$, the cyclization of 3-methoxyanilinederived substrate $7 \mathbf{r}$ being conducted in TFE did not lead to a full conversion within $12 \mathrm{~h}$ at $\mathrm{rt}$ (Table 4, entry 1). Switching to branched fluorinated solvents led to a faster conversion of $7 \mathbf{r}$ simultaneously suppressing the competing alkoxylation (Table 4, entries 2-4). Nonetheless, the transformation of $7 \mathbf{r}$ was further complicated by the fact that the resulting cyclized products $8 \mathbf{r}$ and 8r' were essentially inseparable. The best $\mathbf{8 r} / \mathbf{8 r}$ ' ratio could be obtained using hexafluoro-2-methylpropan-2-ol while the best overall yield was obtained using HFIP (Table 4, entry 2 versus entry 4).

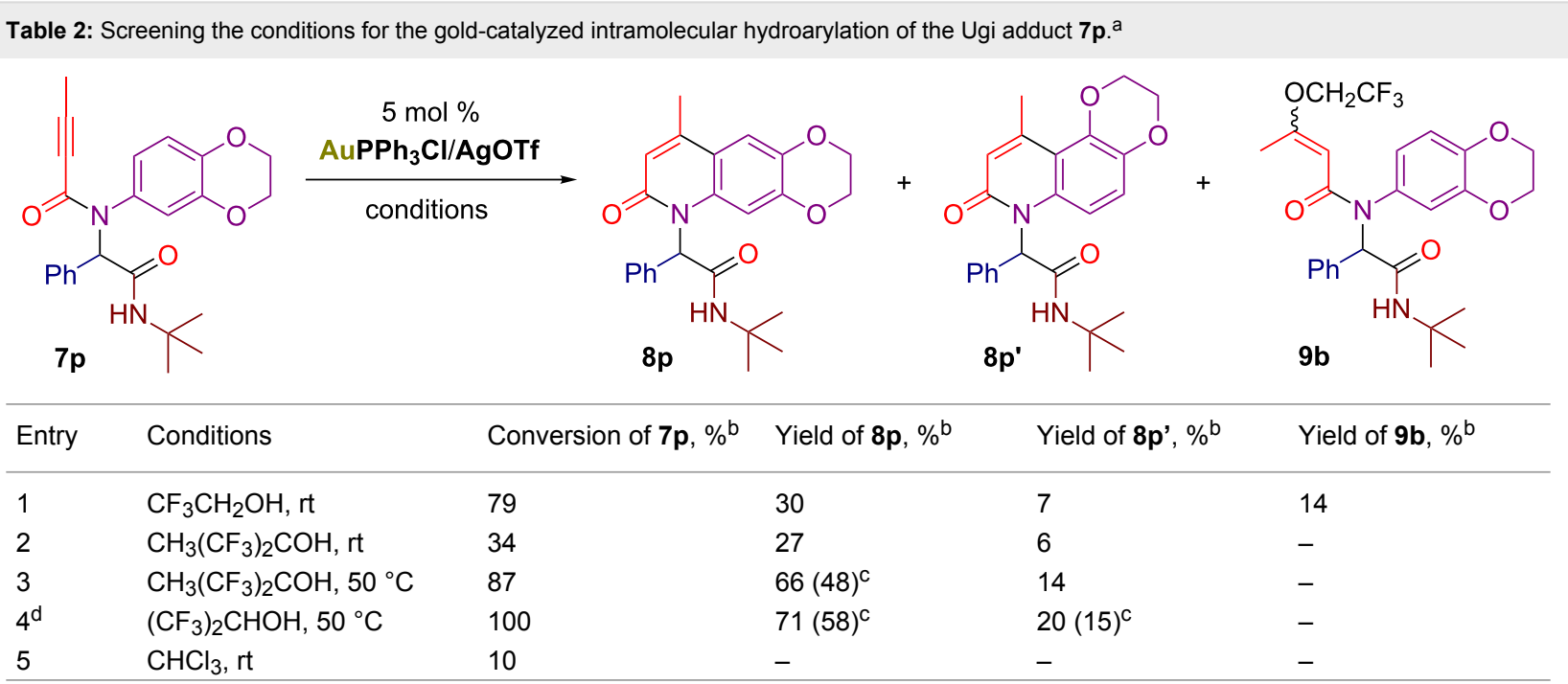

a Reaction conditions: $7 \mathrm{p}(0.2 \mathrm{mmol})$, solvent $(2 \mathrm{~mL}), 12 \mathrm{~h}$, under air. ${ }^{b}$ Determined by ${ }^{1} \mathrm{H}$ NMR using $3,4,5$-trimethoxybenzaldehyde as an internal standard. CIsolated yields for $0.3 \mathrm{mmol}$ scale reactions are given in parentheses. ${ }^{\mathrm{d}}$ The reaction was conducted for $20 \mathrm{~h}$. 
Table 3: Screening the conditions for the gold-catalyzed intramolecular hydroarylation of the Ugi adduct $\mathbf{7 q}{ }^{\text {a }}$<smiles>CC#CC(=O)N(c1ccc(Br)c(OC)c1)C(C(=O)NC(C)(C)C)c1ccccc1</smiles><smiles>COc1cc2c(cc1Br)c(C)cc(=O)n2C(C(=O)NC(C)(C)C)c1ccccc1</smiles><smiles>COc1cc(N(C(=O)/C=C(\C)C(F)(F)F)C(C(=O)NC(C)(C)C)c2ccccc2)ccc1Br</smiles>

\begin{tabular}{|c|c|c|c|c|c|}
\hline Entry & Conditions & Conversion of $\mathbf{7 q}, \%^{b}$ & Yield of $\mathbf{8 q}, \%^{b}$ & Yield of $\mathbf{8} \mathbf{q}^{\prime}, \%^{b}$ & Yield of $9 c, \%^{b}$ \\
\hline 1 & $\mathrm{CF}_{3} \mathrm{CH}_{2} \mathrm{OH}, \mathrm{rt}$ & 28 & 4 & 1 & 11 \\
\hline 2 & $\mathrm{CF}_{3} \mathrm{CH}_{2} \mathrm{OH}, 50^{\circ} \mathrm{C}$ & 58 & 8 & 2 & $22(18)^{c}$ \\
\hline 3 & $\left(\mathrm{CF}_{3}\right)_{2} \mathrm{CHOH}, \mathrm{rt}$ & 12 & 7 & 1 & - \\
\hline 4 & $\left(\mathrm{CF}_{3}\right)_{2} \mathrm{CHOH}, 50^{\circ} \mathrm{C}$ & 24 & 14 & 4 & - \\
\hline $5^{d}$ & $\left(\mathrm{CF}_{3}\right)_{2} \mathrm{CHOH}, 50^{\circ} \mathrm{C}$ & 51 & 40 & 6 & - \\
\hline $6^{e}$ & $\left(\mathrm{CF}_{3}\right)_{2} \mathrm{CHOH}, 50^{\circ} \mathrm{C}$ & 100 & $76(75)^{\mathrm{c}}$ & 18 & - \\
\hline
\end{tabular}

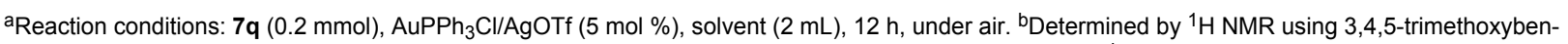
zaldehyde as an internal standard. Clsolated yields for $0.4 \mathrm{mmol}$ scale reactions are given in parentheses. ${ }^{\mathrm{d}}$ The reaction was conducted for $24 \mathrm{~h}$ using $10 \mathrm{~mol} \%$ of $\mathrm{AuPPh}_{3} \mathrm{Cl} / \mathrm{AgOTf}$ catalyst. ${ }^{\mathrm{e}}$ The reaction was conducted for $30 \mathrm{~h}$ using $20 \mathrm{~mol} \%$ of $\mathrm{AuPPh}_{3} \mathrm{Cl} / \mathrm{AgOTf}_{\mathrm{g}}$ catalyst.

Table 4: Screening the conditions for the gold-catalyzed intramolecular hydroarylation of the Ugi adduct $7 r^{\text {a }}$

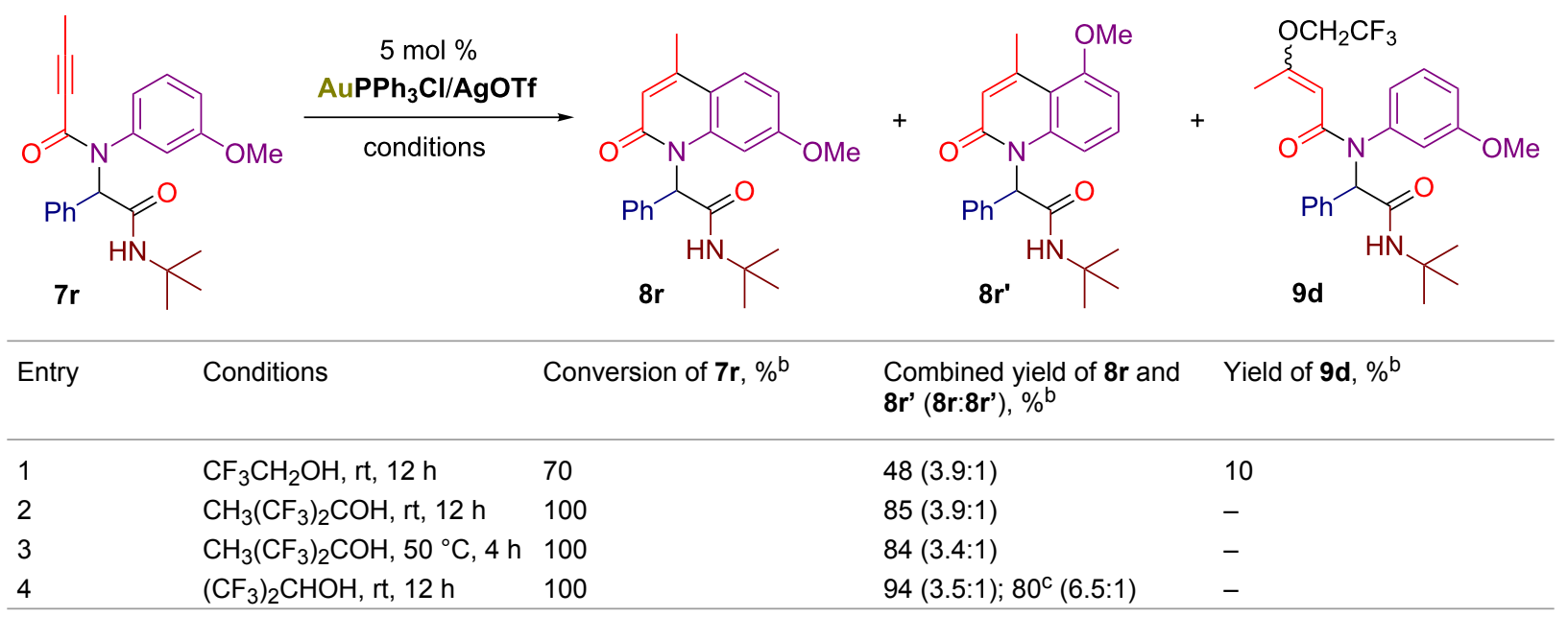

aReaction conditions: $7 \mathrm{r}(0.15 \mathrm{mmol})$, solvent $(1.5 \mathrm{~mL})$, under air. ${ }^{b}$ Determined by ${ }^{1} \mathrm{H}$ NMR using 3,4,5-trimethoxybenzaldehyde as an internal standard. ${ }^{c}$ Combined yield for a $0.4 \mathrm{mmol}$ scale reaction obtained after column chromatography.

\section{Conclusion}

We have elaborated a fast and diversity-oriented approach towards 2-quinolones bearing a branched substituent on the nitrogen atom. The strategy relies on the application of a fourcomponent Ugi reaction followed by a gold-catalyzed intramolecular alkyne hydroarylation. The developed process has a broad scope and simple reaction settings. Another important highlight of the developed process is that it strongly benefits from the employment of fluorinated alcohols as environmentally benign solvents.

\section{Supporting Information}

\section{Supporting Information File 1}

Full experimental procedures and spectroscopic characterizations, as well as the copies of ${ }^{1} \mathrm{H}$ and ${ }^{13} \mathrm{C}$ NMR spectra of Ugi products 7 and final 2-quinolones 8.

[https://www.beilstein-journals.org/bjoc/content/ supplementary/1860-5397-14-234-S1.pdf] 


\section{Acknowledgements}

This work was supported by the start-up fund from Soochow University (grant Q410900714), National Natural Science Foundation of China (grant 21650110445), Natural Science Foundation of Jiangsu Province of China (grants BK20160310and BK20150317), the Priority Academic Program Development of Jiangsu Higher Education Institutions (PAPD) and the project of scientific and technologic infrastructure of Suzhou (grant SZS201708). A. A. N. is grateful to the European Community Mobility Programme "Erasmus Mundus Action 2, Strand 1" for providing a doctoral scholarship. E. V. V. d. E. acknowledges the support of RUDN University Program 5-100.

\section{ORCID ${ }^{\circledR}$ iDs}

Vsevolod A. Peshkov - https://orcid.org/0000-0001-6649-2384

\section{References}

1. Heeb, S.; Fletcher, M. P.; Chhabra, S. R.; Diggle, S. P.; Williams, P.; Cámara, M. FEMS Microbiol. Rev. 2011, 35, 247-274. doi:10.1111/j.1574-6976.2010.00247.x

2. Michael, J. P. Nat. Prod. Rep. 2008, 25, 166-187. doi:10.1039/b612168n

3. Andersson, M. I.; MacGowan, A. P. J. Antimicrob. Chemother. 2003, 51 (Suppl. 1), 1-11. doi:10.1093/jac/dkg212

4. Tedesco, R.; Chai, D.; Darcy, M. G.; Dhanak, D.; Fitch, D. M.; Gates, A.; Johnston, V. K.; Keenan, R. M.; Lin-Goerke, J.; Sarisky, R. T.; Shaw, A. N.; Valko, K. L.; Wiggall, K. J.; Zimmerman, M. N.; Duffy, K. J. Bioorg. Med. Chem. Lett. 2009, 19, 4354-4358. doi:10.1016/j.bmcl.2009.05.080

5. Cheng, P.; Zhang, Q.; Ma, Y.-B.; Jiang, Z.-Y.; Zhang, X.-M.; Zhang, F.-X.; Chen, J.-J. Bioorg. Med. Chem. Lett. 2008, 18, 3787-3789. doi:10.1016/j.bmcl.2008.05.065

6. Guo, R.-H.; Zhang, Q.; Ma, Y.-B.; Luo, J.; Geng, C.-A.; Wang, L.-J.; Zhang, X.-M.; Zhou, J.; Jiang, Z.-Y.; Chen, J.-J. Eur. J. Med. Chem. 2011, 46, 307-319. doi:10.1016/j.ejmech.2010.11.019

7. Curreli, F.; Zhang, H.; Zhang, X.; Pyatkin, I.; Victor, Z.; Altieri, A.; Debnath, A. K. Bioorg. Med. Chem. 2011, 19, 77-90. doi:10.1016/j.bmc.2010.11.045

8. Naik, M.; Humnabadkar, V.; Tantry, S. J.; Panda, M.; Narayan, A.; Guptha, S.; Panduga, V.; Manjrekar, P.; Jena, L. k.; Koushik, K.; Shanbhag, G.; Jatheendranath, S.; Manjunatha, M. R.; Gorai, G.; Bathula, C.; Rudrapatna, S.; Achar, V.; Sharma, S.; Ambady, A.; Hegde, N.; Mahadevaswamy, J.; Kaur, P.; Sambandamurthy, V. K.; Awasthy, D.; Narayan, C.; Ravishankar, S.; Madhavapeddi, P.; Reddy, J.; Prabhakar, K.; Saralaya, R.; Chatterji, M.; Whiteaker, J.; McLaughlin, B.; Chiarelli, L. R.; Riccardi, G.; Pasca, M. R.; Binda, C.; Neres, J.; Dhar, N.; Signorino-Gelo, F.; McKinney, J. D.; Ramachandran, V.; Shandil, R.; Tommasi, R.; Iyer, P. S.; Narayanan, S.; Hosagrahara, V.; Kavanagh, S.; Dinesh, N.; Ghorpade, S. R. J. Med. Chem. 2014, 57, 5419-5434. doi:10.1021/jm5005978

9. Hassanin, H. M.; El-edfawy, S. M. Heterocycles 2012, 85, 2421-2436. doi:10.3987/com-12-12523

10. Kraus, J. M.; Verlinde, C. L. M. J.; Karimi, M.; Lepesheva, G. I.; Gelb, M. H.; Buckner, F. S. J. Med. Chem. 2009, 52, 1639-1647. doi:10.1021/jm801313t
11. Kraus, J. M.; Tatipaka, H. B.; McGuffin, S. A.; Chennamaneni, N. K.; Karimi, M.; Arif, J.; Verlinde, C. L. M. J.; Buckner, F. S.; Gelb, M. H. J. Med. Chem. 2010, 53, 3887-3898. doi:10.1021/jm9013136

12. Clark, P. G. K.; Vieira, L. C. C.; Tallant, C.; Fedorov, O.; Singleton, D. C.; Rogers, C. M.; Monteiro, O. P.; Bennett, J. M.; Baronio, R.; Müller, S.; Daniels, D. L.; Méndez, J.; Knapp, S.; Brennan, P. E.; Dixon, D. J. Angew. Chem., Int. Ed. 2015, 54, 6217-6221. doi:10.1002/anie.201501394

13. Upadhyay, K. D.; Dodia, N. M.; Khunt, R. C.; Chaniara, R. S.; Shah, A. K. ACS Med. Chem. Lett. 2018, 9, 283-288. doi:10.1021/acsmedchemlett.7b00545

14. Alsina, M.; Fonseca, R.; Wilson, E. F.; Belle, A. N.; Gerbino, E.; Price-Troska, T.; Overton, R. M.; Ahmann, G.; Bruzek, L. M.; Adjei, A. A.; Kaufmann, S. H.; Wright, J. J.; Sullivan, D.; Djulbegovic, B.; Cantor, A. B.; Greipp, P. R.; Dalton, W. S.; Sebti, S. M. Blood 2004, 103, 3271-3277. doi:10.1182/blood-2003-08-2764

15. Sparano, J. A.; Moulder, S.; Kazi, A.; Coppola, D.; Negassa, A.; Vahdat, L.; Li, T.; Pellegrino, C.; Fineberg, S.; Munster, P.; Malafa, M.; Lee, D.; Hoschander, S.; Hopkins, U.; Hershman, D.; Wright, J. J.; Kleer, C.; Merajver, S.; Sebti, S. M. Clin. Cancer Res. 2009, 15, 2942-2948. doi:10.1158/1078-0432.ccr-08-2658

16. Ni, Z.-J.; Barsanti, P.; Brammeier, N.; Diebes, A.; Poon, D. J.; Ng, S.; Pecchi, S.; Pfister, K.; Renhowe, P. A.; Ramurthy, S.; Wagman, A. S.; Bussiere, D. E.; Le, V.; Zhou, Y.; Jansen, J. M.; Ma, S.; Gesner, T. G. Bioorg. Med. Chem. Lett. 2006, 16, 3121-3124. doi:10.1016/j.bmcl.2006.03.059

17. Claassen, G.; Brin, E.; Crogan-Grundy, C.; Vaillancourt, M. T.; Zhang, H. Z.; Cai, S. X.; Drewe, J.; Tseng, B.; Kasibhatla, S. Cancer Lett. 2009, 274, 243-249. doi:10.1016/j.canlet.2008.09.032

18. Maiti, A.; Reddy, P. V. N.; Sturdy, M.; Marler, L.; Pegan, S. D.; Mesecar, A. D.; Pezzuto, J. M.; Cushman, M. J. Med. Chem. 2009, 52, 1873-1884. doi:10.1021/jm801335z

19. Tang, Q.; Zhai, X.; Tu, Y.; Wang, P.; Wang, L.; Wu, C.; Wang, W.; Xie, H.; Gong, P.; Zheng, P. Bioorg. Med. Chem. Lett. 2016, 26, 1794-1798. doi:10.1016/j.bmcl.2016.02.037

20. Bonnefous, C.; Payne, J. E.; Roppe, J.; Zhuang, H.; Chen, X.; Symons, K. T.; Nguyen, P. M.; Sablad, M.; Rozenkrants, N.; Zhang, Y.; Wang, L.; Severance, D.; Walsh, J. P.; Yazdani, N.; Shiau, A. K.; Noble, S. A.; Rix, P.; Rao, T. S.; Hassig, C. A.; Smith, N. D. J. Med. Chem. 2009, 52, 3047-3062. doi:10.1021/jm900173b

21. Payne, J. E.; Bonnefous, C.; Symons, K. T.; Nguyen, P. M.; Sablad, M.; Rozenkrants, N.; Zhang, Y.; Wang, L.; Yazdani, N.; Shiau, A. K.; Noble, S. A.; Rix, P.; Rao, T. S.; Hassig, C. A.; Smith, N. D. J. Med. Chem. 2010, 53, 7739-7755. doi:10.1021/jm100828n

22. Durón, S. G.; Lindstrom, A.; Bonnefous, C.; Zhang, H.; Chen, X.; Symons, K. T.; Sablad, M.; Rozenkrants, N.; Zhang, Y.; Wang, L.; Yazdani, N.; Shiau, A. K.; Noble, S. A.; Rix, P.; Rao, T. S.; Hassig, C. A.; Smith, N. D. Bioorg. Med. Chem. Lett. 2012, 22, 1237-1241. doi:10.1016/j.bmcl.2011.11.073

23. Hewawasam, P.; Fan, W.; Ding, M.; Flint, K.; Cook, D.; Goggins, G. D.; Myers, R. A.; Gribkoff, V. K.; Boissard, C. G.; Dworetzky, S. I.; Starrett, J. E., Jr.; Lodge, N. J. J. Med. Chem. 2003, 46, 2819-2822. doi:10.1021/jm030005h

24. Huang, C.-C.; Chang, N.-C. Org. Lett. 2008, 10, 673-676. doi:10.1021/ol7030312

25. Glasnov, T. N.; Stadlbauer, W.; Kappe, C. O. J. Org. Chem. 2005, 70, 3864-3870. doi:10.1021/jo0502549

26. Chen, X.; Cui, X.; Wu, Y. Org. Lett. 2016, 18, 2411-2414. doi:10.1021/acs.orglett.6b00923 
27. Guo, T. Tetrahedron Lett. 2016, 57, 5837-5840. doi:10.1016/j.tetlet.2016.11.056

28. Guo, T.; Wang, H. Synlett 2017, 28, 1845-1851. doi:10.1055/s-0036-1588829

29. Guo, T.; Wei, X.-N.; Wang, H.-Y.; Zhao, B. Synth. Commun. 2018, 48, 761-767. doi:10.1080/00397911.2017.1422519

30. Ishida, T.; Kikuchi, S.; Yamada, T. Org. Lett. 2013, 15, 3710-3713. doi:10.1021/ol401571r

31. Zhang, J.; Han, X.; Lu, X. Synlett 2015, 26, 1744-1748. doi:10.1055/s-0034-1380751

32. Murayama, T.; Shibuya, M.; Yamamoto, Y. J. Org. Chem. 2016, 81 11940-11949. doi:10.1021/acs.joc.6b02626

33. Liu, L.; Lu, H.; Wang, H.; Yang, C.; Zhang, X.; Zhang-Negrerie, D.; Du, Y.; Zhao, K. Org. Lett. 2013, 15, 2906-2909. doi:10.1021/ol400743r

34. Mai, W.-P.; Sun, G.-C.; Wang, J.-T.; Song, G.; Mao, P.; Yang, L.-R.; Yuan, J.-W.; Xiao, Y.-M.; Qu, L.-B. J. Org. Chem. 2014, 79, 8094-8102. doi:10.1021/jo501301t

35. Manikandan, R.; Jeganmohan, M. Org. Lett. 2014, 16, 3568-3571. doi:10.1021/ol501548e

36. Wu, J.; Xiang, S.; Zeng, J.; Leow, M.; Liu, X.-W. Org. Lett. 2015, 17, 222-225. doi:10.1021/ol503292p

37. Dorel, R.; Echavarren, A. M. Chem. Rev. 2015, 115, 9028-9072. doi:10.1021/cr500691k

38. Boyarskiy, V. P.; Ryabukhin, D. S.; Bokach, N. A.; Vasilyev, A. V. Chem. Rev. 2016, 116, 5894-5986. doi:10.1021/acs.chemrev.5b00514

39. Iwai, I.; Hiraoka, T. Chem. Pharm. Bull. 1963, 11, 638-643. doi:10.1248/cpb.11.638

40. Koltunov, K. Y.; Walspurger, S.; Sommer, J. Eur. J. Org. Chem. 2004, 4039-4047. doi:10.1002/ejoc.200400313

41. Ryabukhin, D. S.; Gurskaya, L. Y.; Fukin, G. K.; Vasilyev, A. V. Tetrahedron 2014, 70, 6428-6443. doi:10.1016/j.tet.2014.07.028

42. Jia, C.; Piao, D.; Kitamura, T.; Fujiwara, Y. J. Org. Chem. 2000, 65, 7516-7522. doi:10.1021/jo000861q

43. Song, C. E.; Jung, D.-u.; Choung, S. Y.; Roh, E. J.; Lee, S.-g. Angew. Chem., Int. Ed. 2004, 43, 6183-6185. doi:10.1002/anie.200460292

44. Vacala, T.; Bejcek, L. P.; Williams, C. G.; Williamson, A. C.; Vadola, P. A. J. Org. Chem. 2017, 82, 2558-2569. doi:10.1021/acs.joc.6b02984

45. Shibuya, T.; Shibata, Y.; Noguchi, K.; Tanaka, K. Angew. Chem., Int. Ed. 2011, 50, 3963-3967. doi:10.1002/anie.201100152

46. Shibuya, T.; Nakamura, K.; Tanaka, K. Beilstein J. Org. Chem. 2011, 7, 944-950. doi:10.3762/bjoc.7.105

47. Banfi, L.; Basso, A.; Riva, R. Top. Heterocycl. Chem. 2010, 23, 1-39. doi:10.1007/7081_2009_23

48. Sharma, U. K.; Sharma, N.; Vachhani, D. D.; Van der Eycken, E. V. Chem. Soc. Rev. 2015, 44, 1836-1860. doi:10.1039/c4cs00253a

49. Li, X.; Jia, X.; Yin, L. Chin. J. Org. Chem. 2017, 37, 2237-2249. doi:10.6023/cjoc201704026

50. Liu, C.; Wang, G.; Wang, Y.; Van Hecke, K.; Pereshivko, O. P.; Peshkov, V. A. Tetrahedron Lett. 2018, 59, 1823-1827. doi:10.1016/j.tetlet.2018.03.079

51. Vachhani, D. D.; Kumar, A.; Modha, S. G.; Sharma, S. K.; Parmar, V. S.; Van der Eycken, E. V. Synthesis 2015, 47, 1337-1347. doi:10.1055/s-0034-1379894

52. Li, Z.; Kumar, A.; Vachhani, D. D.; Sharma, S. K.; Parmar, V. S.; Van der Eycken, E. V. Eur. J. Org. Chem. 2014, 2084-2091. doi:10.1002/ejoc.201301507
53. Kumar, A.; Li, Z.; Sharma, S. K.; Parmar, V. S.; Van der Eycken, E. V. Chem. Commun. 2013, 49, 6803-6805. doi:10.1039/c3cc42704h

54. He, Y.; Li, Z.; Robeyns, K.; Van Meervelt, L.; Van der Eycken, E. V. Angew. Chem., Int. Ed. 2018, 57, 272-276.

doi:10.1002/anie.201710592

55. Vacala, T. L.; Carlson, P. R.; Arreola-Hester, A.; Williams, C. G.; Makhoul, E. W.; Vadola, P. A. J. Org. Chem. 2018, 83, 1493-1501. doi:10.1021/acs.joc.7b03125

56. He, Y.; Li, Z.; Tian, G.; Song, L.; Van Meervelt, L.; Van der Eycken, E. V. Chem. Commun. 2017, 53, 6413-6416. doi:10.1039/c7cc03152a

57. Kumar, A.; Vachhani, D. D.; Modha, S. G.; Sharma, S. K.; Parmar, V. S.; Van der Eycken, E. V. Beilstein J. Org. Chem. 2013, 9 , 2097-2102. doi:10.3762/bjoc.9.246

58. Modha, S. G.; Kumar, A.; Vachhani, D. D.; Jacobs, J.; Sharma, S. K.; Parmar, V. S.; Van Meervelt, L.; Van der Eycken, E. V. Angew. Chem., Int. Ed. 2012, 51, 9572-9575. doi:10.1002/anie.201205052

59. Nechaev, A. A.; Van Hecke, K.; Zaman, M.; Kashtanov, S.; Ungur, L.; Pereshivko, O. P.; Peshkov, V. A.; Van der Eycken, E. V. J. Org. Chem. 2018, 83, 8170-8182. doi:10.1021/acs.joc.8b00953

60. Rasouli, M. A.; Mahdavi, M.; Saeedi, M.; Ranjbar, P. R.; Shafiee, A.; Foroumadi, A. J. Heterocycl. Chem. 2015, 52, 386-391. doi:10.1002/jhet.2053

61. Gordon, C. P.; Young, K. A.; Hizartzidis, L.; Deane, F. M.; McCluskey, A. Org. Biomol. Chem. 2011, 9, 1419-1428. doi:10.1039/c0ob00029a

62. Marcaccini, S.; Pepino, R.; Pozo, M. C.; Basurto, S.; Garcia-Valverde, M.; Torroba, T. Tetrahedron Lett. 2004, 45, 3999-4001. doi:10.1016/j.tetlet.2004.03.184

63. Dandia, A.; Singh, R.; Joshi, J.; Kumari, S. Mini-Rev. Org. Chem. 2014, 11, 462-476. doi:10.2174/1570193×1104140926170237

\section{License and Terms}

This is an Open Access article under the terms of the Creative Commons Attribution License (http://creativecommons.org/licenses/by/4.0). Please note that the reuse, redistribution and reproduction in particular requires that the authors and source are credited.

The license is subject to the Beilstein Journal of Organic Chemistry terms and conditions: (https://www.beilstein-journals.org/bjoc)

The definitive version of this article is the electronic one which can be found at: doi:10.3762/bjoc. 14.234 